\title{
Theoretical Analysis of Stability of Axially SYMMETRIC RotaTing OBJECTS WiTH Regard to Orienting APPles
}

\author{
P. Narayanan, A. M. Lefcourt, U. Tasch, R. Rostamian, A. Grinblat, M. S. Kim
}

\begin{abstract}
Inspection using machine vision offers the potential for improved food safety and quality. However, effectiveness of fruit inspection has been limited by the difficulty of appropriately orienting fruit for imaging. Commercial orientation systems have had limited impact due to mechanical complexity, cost, error, or some combination thereof. Preliminary tests demonstrated that apples could be oriented by rolling them down a track consisting of two parallel rails. After achieving sufficient angular velocity, the apples moved to an orientation where the stem/calyx axis was perpendicular to the direction of travel and parallel to the plane of the track. The purpose of the current study was to examine whether this orientation phenomenon could be explained in terms of the inertial characteristics of axially symmetric objects. Rotation of a free body around an axis of axial symmetry was found to be stable, while rotation around an axis perpendicular to this symmetric axis was not. Furthermore, comparisons of action values for two different mathematical models of apples indicate that inertial characteristics can be used to orient apples. The critical assumption for this analysis was that for the same object traveling the same path with different initial orientations, lower action values represent preferred motions. This study introduces the novel use of action integrals to examine stability, and the results provide testable predictions that round apples as compared to elongated or squat apples and larger elongated apples will need to travel farther before orientation is favored.
\end{abstract}

Keywords. Apples, Calyx, Machine vision, Orientation, Physical properties, Stem.

$\mathrm{M}$ achine vision is increasingly used for automated inspection of agricultural commodities (Brosnan and Sun, 2004; Chen et al., 2002). Commercial systems are available that allow fruit to be sorted by size, shape, and color. Research results suggest that it is feasible to use machine vision systems to inspect fruit for quality problems (Bennedsen and Peterson, 2005; Brosnan and Sun, 2004; Kleynen et al., 2005; Mehl et al., 2004; Throop et al., 2005) and for fecal contamination (Kim et al., 2002; Lefcourt et al., 2003, 2005). Currently, sorting of fruits for surface defects is mainly done by manual inspection (Bennedsen and Peterson, 2005), and no commercial system is available for detecting fecal contamination. Researchers have developed computerimaging methods for detecting bruised and diseased fruit (Brown et al., 1974; Li et al., 2002; Kleynen et al., 2005;

Submitted for review in May 2006 as manuscript number FPE 6485; approved for publication by the Food \& Process Engineering Institute Division of ASABE in July 2008.

The authors are Priya Narayanan, Graduate Student, Department of Mechanical Engineering, University of Maryland, Baltimore County (UMBC), Baltimore, Maryland; Alan Marc Lefcourt, ASABE Member Engineer, Research Biomedical Engineer, USDA-ARS Henry A. Wallace Beltsville Agricultural Research Center, Beltsville, Maryland; Uri Tasch, Professor, Department of Mechanical Engineering, Rouben Rostamian, Professor, Department of Mathematics and Statistics, and Abraham Grinblat, Research Associate, Department of Mechanical Engineering, UMBC, Baltimore, Maryland; and Moon S. Kim, Research Physical Scientist, USDA-ARS Henry A. Wallace Beltsville Agricultural Research Center, Beltsville, Maryland. Corresponding author: Uri Tasch, Department of Mechanical Engineering, UMBC, 1000 Hilltop Circle Baltimore, MD 21250; phone: 410-455-3305; fax: 410-455-1052; email: tasch@umbc.edu.
Throop et al., 2005) and feces-contaminated fruit (Kim et al., 2002; Lefcourt et al., 2003, 2005). However, it has proven difficult to develop efficient algorithms to discriminate between bruise sites and the stem or calyx. Complex image processing algorithms have been written in order to detect the stem and the calyx region; however, these methods usually are expensive and time-consuming (Bennedsen and Peterson, 2004; Kleynen et al., 2005; Penman, 2001; Throop et al., 2005). Alternatively, a secondary imaging system can be used to identify the stem and calyx regions, again a costly option (Wen and Tao, 2000). One solution to this problem is to orient the fruit prior to imaging, so that the location of the stem and calyx regions are known and can be excluded from images if desired. For contamination detection, control of the location of the stem and calyx is particularly important, as these areas are concave and screening $100 \%$ of the surface of a fruit for contamination requires imaging inside these regions.

Over the last 50 years, a number of mechanical systems to orient fruit have been developed; however, commercial impact has been limited due to mechanical complexity, cost, error, or some combination thereof. The physical properties of fruit have been examined in detail (Megilley et al., 1968; Rigney et al., 1996; Stout et al., 1971; Throop et al., 2001), and a number of attempts have been made to use rotation to orient fruit. One such device used rollers with conical surfaces sloping towards that center so that the fruit are "automatically oriented to stand up and rotate in wheel-like formation" (Brown et al., 1988). Another system was designed for orienting peaches based on the concept of rolling a peach along an upward-inclined runway (Brusewitz et al., 1995). A paddle driven by a motor was used to push 


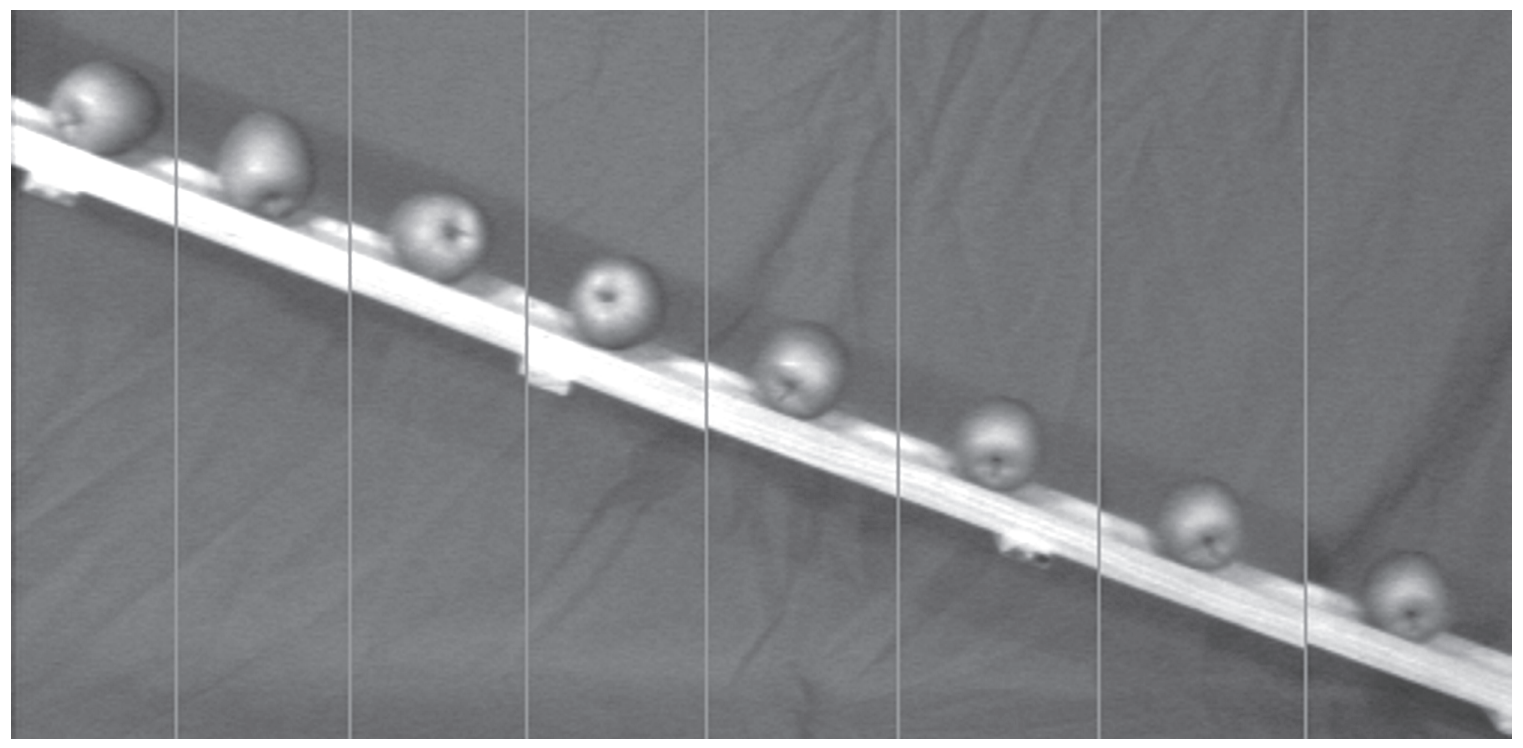

Figure 1. Position and orientation of an apple as it rolls down a test track using a collage of sequential images taken at $0.1 \mathrm{~s}$ intervals. It can be seen that the apple initially had no specific orientation, but that towards the end the apple oriented itself such that the stem/calyx axis was perpendicular to the direction of travel.

peaches along the runway. It was observed that orientation performance was dependent on the diameter-to-height ratio of a peach (Rigney et al., 1996). A device with multiple rollers was developed to orient apples such that "the apple has a tendency to seek a position where its largest diameter engages the drive roller with the axis of rotation for the fruit lying along its shortest dimension" (Throop et al., 1999; Throop et al., 2001). More recently, the concave shapes of the stem and calyx regions were used to orient apples (Throop et al., 2005). Although these studies did not specifically examine the theoretical basis of the orientation process, the primary factors responsible for orientation were the physical shape of the fruit and the effects of gravity.

In contrast, we discovered that fruit could be oriented based on inertial properties (Narayanan et al., 2007; Tasch et al., 2006). This approach involves imparting angular momentum to the fruit and using inertial properties to orient the fruit along the stem/calyx axis. To test this concept, a test track was constructed, and apples of various shapes were rolled down the track (fig. 1). It was observed that as angular velocity increased, the apples generally moved to an oriented position in which the stem-calyx axis was perpendicular to the direction of travel and parallel to the plane of the tracks.

The goal of the current study was to determine if an axially symmetric object is theoretically more stable when it rotates around the symmetric axis. In this regard, two independent tests were performed. In the first test, analysis of stability was undertaken for a freely rotating rigid object using the method of small perturbation. In the second test, the dynamics of a rigid object rolling on a flat plane was studied using the action integral.

\section{Materials and Methods}

\section{Rotational StabiLITy}

The dynamics of a freely rotating object can be described using the Euler equations (Marion, 1965). These equations can then be used to examine the stability of the rotating object using the small perturbation method. Assuming the moments (system inputs) to be zero, the stability criterion for the rotational motion of a generalized object about a principal axis has been derived (Greenwood, 1965; Marion, 1965). This criterion was used to derive stability criteria for an axially symmetric object, and the stability for rotation about the axially symmetric axis and about an axis perpendicular to this axis was determined.

\section{ACTION INTEGRAL}

The classical definition of minimum action states: "Of all the possible paths along which a dynamical system may move from one point to another within a specified time interval, the actual path followed is that which minimizes the action." Action $(S)$ can be defined as:

$$
S=\int_{0}^{t} L d t
$$

where the Lagrangian $(L)$ equals the kinetic energy $(K E)$ minus the potential energy $(P E)$, and $t$ is the time at the end point of the path, respectively (Meirovitch, 1988). In this study, action values were calculated for an object moving on a fixed path given different initial orientations. It was assumed that conditions that produce the lowest relative action values represent preferred motions. A corollary of this assumption is that the magnitude of differences between calculated action values for different initial orientations is indicative of the degree of relative preference. To calculate $S$, the differential equations $L=\frac{d S}{d t}$ (from eq. 1) and $\omega=\frac{d \theta}{d t}$ with initial condition $\theta_{0}$ were numerically solved, where $\omega$ is the angular velocity of the object, and $\theta$ is the angle of rotation of the object. Calculations were done using Maple software (Maplesoft, Ontario, Canada).

For simplicity, apples were initially modeled as ellipsoids for action integral analyses. It was assumed that the ellipsoids roll in a straight line along a level plane, linear acceleration of the ellipsoids is constant, and there is no slippage. To facilitate 
comparisons, the term "action difference" was defined as the difference between the action values for an object given two different initial orientations. This measure was used to examine the potential influence of physical characteristics of "apples" on the orientation phenomena. Most analyses were replicated with the ellipsoid models of apples replaced by spindle torus models, which more accurately represent the shapes of different varieties of apples.

\section{Results AND Discussion}

\section{Rotational Stability}

Consider a rigid body of arbitrary shape with distinct principal moments of inertia $I_{1}, I_{2}, I_{3}$ rotating with a constant angular velocity $\omega_{1}$ about the axis associated with the moment of inertia $I_{1}$. The angular velocity vector is $\left(\omega_{1}, 0\right.$, $0)$ when expressed in coordinate axes aligned with the principal directions of moment of inertia.

If the system is given a slight disturbance and then allowed to continue rotating freely, the angular velocity vector changes to $\left(\omega_{1}+\varepsilon, \lambda, \mu\right)$, where the perturbations $\varepsilon, \lambda$, and $\mu$ are much smaller in magnitude than $\omega_{1}$. After substituting the angular velocities into Euler's equations and then linearizing the equations, the perturbation $\lambda$ satisfies the following differential equation (Greenwood, 1965; Marion, 1965):

$$
\ddot{\lambda}+\frac{\left(I_{1}-I_{3}\right)\left(I_{1}-I_{2}\right)}{I_{2} I_{3}} \omega_{1}^{2} \lambda=0
$$

If $I_{1}$ is the inertia around a unique axially symmetric axis, then $I_{2}=I_{3}$, thus equation 2 reduces to:

$$
\ddot{\lambda}+\frac{\left(I_{1}-I_{2}\right)^{2}}{I_{2}^{2}} \omega_{1}^{2} \lambda=0
$$

Equation 3 is always stable since:

$$
\frac{\left(I_{1}-I_{2}\right)^{2}}{I_{2}^{2}} \omega_{1}^{2}>0
$$

This demonstrates that rotation of a free body about the axially symmetric axis is always stable. On the other hand,

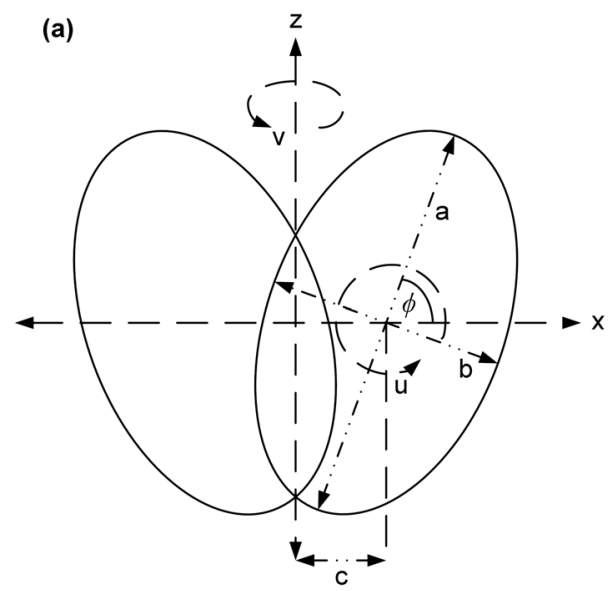

if $I_{2}$ is the axis of symmetry, then $I_{1}=I_{3}$; therefore, equation 2 reduces to:

$$
\ddot{\lambda}=0
$$

Solutions of this equation grow linearly in time. The equations for perturbation $\mu$ are identical to the equations for $\lambda$ in form; therefore, the motion about an axis perpendicular to the axis of symmetry is unstable.

The applicability of these results depends on a number of assumptions. The critical assumption in the stability analysis is axial symmetry. This leads to the condition that the moments of inertia around the principal axes perpendicular to the symmetric axis are equal. However, most apples are not perfectly symmetrical around the stem-calyx axis. This discrepancy could result in instability when additional force and moment components due to the lack of symmetry are introduced.

Another assumption is that the applied external moments are zero, i.e., free body rotation. In reality, as an object rolls down an inclined track, interactions introduce external moments and forces. However, inclusion of these external interactions in calculations would lead to the formulation and necessity of solving complex non-linear equations, which is beyond the scope of this article. Still, the stability of free body rotation about an axially symmetric axis adds credence to the assumption that stability factors are involved in the preliminary observation that apples tend to orient themselves so that they rotate around the stem/calyx axis.

\section{Spindle Torus Apple Model}

To test some of the conclusions regarding stability of free body rotation of objects with axial symmetry, three-dimensional models of apples were developed using a tilted elliptic spindle torus. Models were constructed by revolving an ellipse about an axis that is tilted relative to the ellipse's axes, as shown in figure 2. In a Cartesian coordinate system $(x, y, z)$, the parametric equations of a tilted elliptic spindle torus can be written as:

$$
\begin{aligned}
& x(u, v)=(c+a \cos \varphi \cos u-b \sin \varphi \sin u) \cos v \\
& y(u, v)=(c+a \cos \varphi \cos u-b \sin \varphi \sin u) \sin v
\end{aligned}
$$

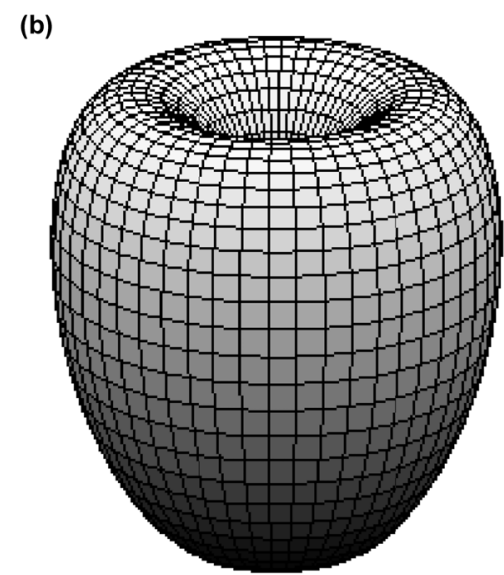

Figure 2. Revolving a tilted ellipse around a center axis to create a tilted elliptic spindle torus. The overlapping region of the rotating ellipse is massless, and thus the apple has a hollow core. 


$$
z(u, v)=(a \sin \varphi \cos u+b \cos \varphi \sin u)
$$

where $c$ is the distance between the center of the ellipse and the axis of revolution ( $z), a$ and $b$ are the semi-major and minor axes of the ellipse, and $\phi$ is the angle between the ellipse's semi-major axis and the $x$-axis. The parameters $u$ and $v$ vary from 0 to $2 \pi$. The geometry of the ellipse and the resulting solid of revolution are shown in figure 2 . The symmetric axis, $z$, corresponds to the stem/calyx axis.

Using equations 6 through 8 and assuming a uniform mass density $\rho$, we can derive the following properties of the solid of revolution:

Volume $(V)$ :

$$
V=2 \pi^{2} a b c
$$

The $z$ coordinate of the center of mass:

$$
z_{C M}=\frac{\left(a^{2}-b^{2}\right) \sin (2 \varphi)}{8 c}
$$

Moment of inertia about the $z$-axis:

$$
I_{z z}=\frac{1}{2} \pi^{2} \rho a b c\left(4 c^{2}+3 a^{2} \cos ^{2} \varphi+3 b^{2} \sin ^{2} \varphi\right)
$$

Moment of inertia about the $x$ or $y$ axes:

$$
\begin{aligned}
I_{x x}=I_{y y} & =\frac{1}{4} \pi^{2} \rho a b c\left[4 c^{2}+\left(2+\cos ^{2} \varphi\right) a^{2}\right. \\
& \left.+\left(2+\sin ^{2} \varphi\right) b^{2}\right]
\end{aligned}
$$

Moment of inertia about an axis perpendicular to the $z$-axis that passes through the center of mass:

$$
\begin{aligned}
& I_{C M}=\frac{1}{32} \pi^{2} \rho \frac{a b}{c}\left[4 c^{2}\left(8 c^{2}+5 a^{2}+5 b^{2}\right)\right. \\
& \left.+4 c^{2}\left(a^{2}-b^{2}\right) \cos 2 \varphi-\left(a^{2}-b^{2}\right)^{2} \sin ^{2} 2 \varphi\right]
\end{aligned}
$$

Equations 9 through 13 were derived assuming that the overlap region of the revolving ellipse is massless. Thus, the resulting "apple" has a hollow core. Additionally, we have assumed that the geometry is such that the two ellipses shown in figure 2 intersect in zero, one, or two points. The case where the ellipses intersect in four points is not covered by these equations. As $I_{x x}=I_{y y} \neq I_{z z}$, equation 2 is applicable, thus verifying that rotation of the spindle torus apple will always be stable on the stem/calyx axis, but unstable on any axis perpendicular to the stem/calyx axis.

\section{EXPANDED APPle MODEL}

The potential usefulness of the spindle torus model of apples was expanded by determining model parameters that correspond to published measurements of physical dimensions of different cultivars of apples. Apples are commonly characterized in terms of length $(L E)$ and diameter $(D)$. While these measurements allow the size of an individual apple to be categorized within a specific cultivar, they cannot be used to differentiate cultivars. Similarly, numerous model parameter sets yield the same length and diameter but result in vastly different shapes. Whitelock et al. (2006) developed a more restrictive measurement set by including a measure of the diameter $(d)$ of apples in the plane perpendicular to the stem/calyx axis and $1.3 \mathrm{~cm}$ from the calyx end. Model parameter sets were developed using this measurement system with one caveat. We measured length in terms of the longest and shortest rib. For an apple that is truly symmetric about the stem/calyx axis, as is the case for a model apple, these measurements are identical. Thus, the rib lengths were averaged to establish the desired length for an apple model.

In order to generate different cultivars of apples, apple model parameters were adjusted to best fit the desired $D, d$, and length to diameter ratio $(L E / D)$ values. The $L E / D$ ratio was used as an optimization criterion instead of $L E$, as this ratio is of critical importance in subsequent stability analyses. The parameters $D, d$, and $L E$ can be obtained for the apple model using the following derivation:

The parametric equation for the two-dimensional cross-section of the apple can be written as:

$$
\begin{aligned}
& x(u)=(c+a \cos \varphi \cos u-b \sin \varphi \sin u) \\
& y(u)=(c+a \cos \varphi \cos u-b \sin \varphi \sin u)
\end{aligned}
$$

The derivative of the parametric equation can be represented as $x^{\prime}(u)=\frac{d x(u)}{d u}$ and $y^{\prime}(u)=\frac{d y(u)}{d u}$.

Maximum diameter $D$ is obtained using the following equation:

$$
D=2 x\left(u_{p}\right)
$$

where $u_{p}$ is equal to the value $u$ (within the range $\frac{3 \pi}{2}$ to $2 \pi$ ) that satisfies the equation $x^{\prime}(u)=0$.

Length $L E$ is obtained using the following equation:

$$
L E=2 y\left(u_{p}\right)
$$

where $u_{p}$ is equal to the value $u$ (within the range 0 to $\frac{\pi}{2}$ ) that satisfies the equation $y^{\prime}(u)=0$.

Diameter $d$ is obtained using the following equation:

$$
d=2 x\left(u_{p}\right)
$$

where $u_{p}$ is equal to the value $u$ (within the range $-\frac{\pi}{2}$ to $\frac{\pi}{2}$ ) that satisfies the equation $y^{\prime}(u)=\frac{L E}{2}-1.3$.

Even with the more restrictive characterization of apples, numerous model parameters sets with different shapes can be produced. It was determined that visualization of theoretically acceptable apple models was necessary to identify the model apple that best resembled the apple cultivar of interest. To facilitate this process, a large dataset with 330,000 records was generated by incrementing values of $a, b, c$, and $\phi$. Ranges for values were obtained by physically examining a number of apples. A selection criterion was generated using sums of squared differences for desired and model $D, d$, and $L E / D$ values. Model apples with low values for the selection criterion were examined by looking at cross-sections of the apples. The apple that best matched the shape of the desired cultivar was selected for 
Table 1. Modification of the table of apple cultivars and measurements in Whitelock et al. (2006) that shows the spindle torus model parameters for apples that best replicate both the original measurement values and apple cultivar shape characteristics. The original values for shortest and longest rib lengths were averaged to determine apple lengths $(L E)$.

\begin{tabular}{|c|c|c|c|c|c|c|c|c|c|}
\hline \multicolumn{2}{|c|}{ Cultivar } & $\mathrm{D}(\mathrm{cm})$ & $\mathrm{d}(\mathrm{cm})$ & $\mathrm{LE}(\mathrm{cm})$ & $\mathrm{LE} / \mathrm{D}$ & $\mathrm{a}(\mathrm{cm})$ & $\mathrm{b}(\mathrm{cm})$ & $\mathrm{c}(\mathrm{cm})$ & $\phi(\mathrm{rad})$ \\
\hline \multirow{2}{*}{$\begin{array}{l}\text { Red Delicious } \\
\quad(\lg -\mathrm{WA})\end{array}$} & Original & 7.79 & 6.75 & 7.88 & 1.01 & & & & \\
\hline & Model & 7.84 & 6.59 & 8.10 & 1.03 & 4.05 & 2.20 & 1.71 & 1.55 \\
\hline \multirow{2}{*}{$\begin{array}{l}\text { Red Delicious } \\
\quad(\lg -\mathrm{WV})\end{array}$} & Original & 8.02 & 7.37 & 6.78 & 0.84 & & & & \\
\hline & Model & 8.27 & 6.99 & 6.83 & 0.83 & 3.43 & 2.21 & 1.90 & 1.45 \\
\hline \multirow{2}{*}{$\begin{array}{l}\text { Rome } \\
(\lg )\end{array}$} & Original & 8.23 & 7.78 & 6.49 & 0.79 & & & & \\
\hline & Model & 8.53 & 7.28 & 6.56 & 0.77 & 3.29 & 2.5 & 1.75 & 1.44 \\
\hline \multirow[t]{2}{*}{ Granny Smith } & Original & 8.09 & 6.84 & 6.91 & 0.85 & & & & \\
\hline & Model & 8.09 & 6.83 & 6.91 & 0.85 & 3.47 & 2.10 & 1.92 & 1.46 \\
\hline \multirow[t]{2}{*}{ Braeburn } & Original & 7.33 & 6.89 & 6.69 & 0.91 & & & & \\
\hline & Model & 7.58 & 6.51 & 6.75 & 0.89 & 3.38 & 1.98 & 1.80 & 1.49 \\
\hline \multirow{2}{*}{$\begin{array}{l}\text { Red Delicious } \\
\text { (sm-WV) }\end{array}$} & Original & 7.46 & 7.04 & 6.17 & 0.83 & & & & \\
\hline & Model & 7.72 & 6.67 & 6.23 & 0.81 & 3.12 & 2.14 & 1.70 & 1.45 \\
\hline \multirow{2}{*}{$\begin{array}{l}\text { Rome } \\
(\mathrm{sm})\end{array}$} & Original & 7.49 & 7.25 & 5.82 & 0.77 & & & & \\
\hline & Model & 7.81 & 6.76 & 5.96 & 0.76 & 3.00 & 2.15 & 1.73 & 1.40 \\
\hline \multirow{2}{*}{$\begin{array}{l}\text { Red Delicious } \\
\quad(\mathrm{sm}-\mathrm{WA})\end{array}$} & Original & 6.97 & 6.57 & 6.26 & 0.89 & & & & \\
\hline & Model & 7.22 & 6.24 & 6.31 & 0.87 & 3.16 & 2.00 & 1.60 & 1.48 \\
\hline \multirow[t]{2}{*}{ Fuji } & Original & 6.82 & 6.65 & 5.75 & 0.84 & & & & \\
\hline & Model & 7.11 & 6.16 & 5.98 & 0.84 & 2.99 & 2.20 & 1.34 & 1.47 \\
\hline
\end{tabular}

further optimization. It was found that $\phi$ was the most important determinant of apple shape. Therefore, the value of $\phi$ was fixed, and the optimal values for $a, b$, and $c$ were obtained using the optimization tool NLPSolve in Maple. By fixing $\phi$, the minimized criterion value was not the lowest value obtainable, but it was the lowest value where the apple model had the shape characteristics of the desired cultivar. If $\phi$ is allowed to vary, then the optimization routine almost always returns the minimum value of $\phi$ allowed. Table 1 reproduces the table of apple cultivars and measurements in Whitelock et al. (2006) and adds parameters for the corresponding optimal apple model.

\section{ACTION InTEGRAL}

\section{Action Integral Using Ellipsoid Model}

Mathematically, an apple can be modeled as an ellipsoid, which can be generated by revolving an ellipse $\left(z^{2} / a^{2}+y^{2} / b^{2}\right.$ $=1$ ) about the $z$-axis (fig. 3 ). In this model, $L E$ corresponds to the distance $2 a$ along the $z$-axis of the ellipsoid, and $D$ corresponds to the distance $2 b$ along an axis perpendicular to the $z$-axis of the ellipsoid, e.g., the $x$-axis. Thus, the stem/ calyx axis corresponds to the axially symmetric $z$-axis of the ellipsoid, and the length-to-diameter ratio $(L E / D)$ of the

\begin{tabular}{ccc}
\multicolumn{3}{c}{$\begin{array}{c}\text { Table 2. Average length }(\boldsymbol{L E}) \text { to diameter }(\boldsymbol{D}) \text { ratio } \\
\text { and average mass of selected cultivars of apples. }\end{array}$} \\
\hline Apple Cultivar & LE/D Ratio & Mass $(\mathrm{g})$ \\
\hline Red Delicious & $0.68-1.01^{[\mathrm{a}]}, 1.1^{[\mathrm{b}]}$ & 215.8 \\
McIntosh & $0.75-0.91^{[\mathrm{a}]}$ & 182.7 \\
Rome & $0.81^{[\mathrm{b}]}$ & 206.2 \\
Granny Smith & $0.91^{[\mathrm{b}]}$ & 201.3 \\
Braeburn & $0.94^{[\mathrm{b}]}$ & 169.9 \\
Fuji & $0.85^{[\mathrm{b}]}$ & 140.1 \\
\hline
\end{tabular}

[a] Stout et al. (1971).

[b] Data taken from Whitelock et al. (2006).

apple corresponds to the $a / b$ ratio of the ellipsoid. From table 2, it can be seen that the $L E / D$ ratio for selected cultivars of apples have ranges of 0.7 to 1.1 , while the average mass ranges from 140 to $215 \mathrm{~g}$. For this study, the $a / b$ ratios of ellipsoids were varied to simulate the range of $L E / D$ ratios. A density of $0.856 \mathrm{~g} \mathrm{~m}^{-3}$ (Whitelock et al., 2006) and a mass of $189 \mathrm{~g}$ were used for a standard ellipsoid model. The masses of ellipsoids were varied as a percentage of this standard mass to encompass the range of values exhibited by apple cultivars.
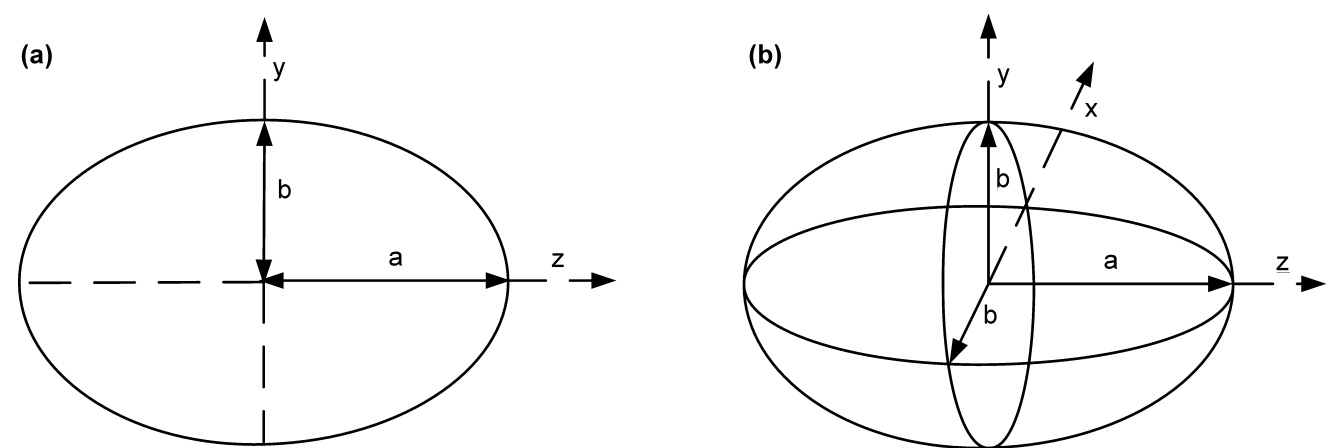

Figure 3. Ellipse (a) with semi-axes $a$ and $b$ that is used to generate an ellipsoid (b), which is obtained by rotating the ellipse around the $z$-axis. Note that the resulting ellipsoid is axially symmetric around the $z$-axis. 

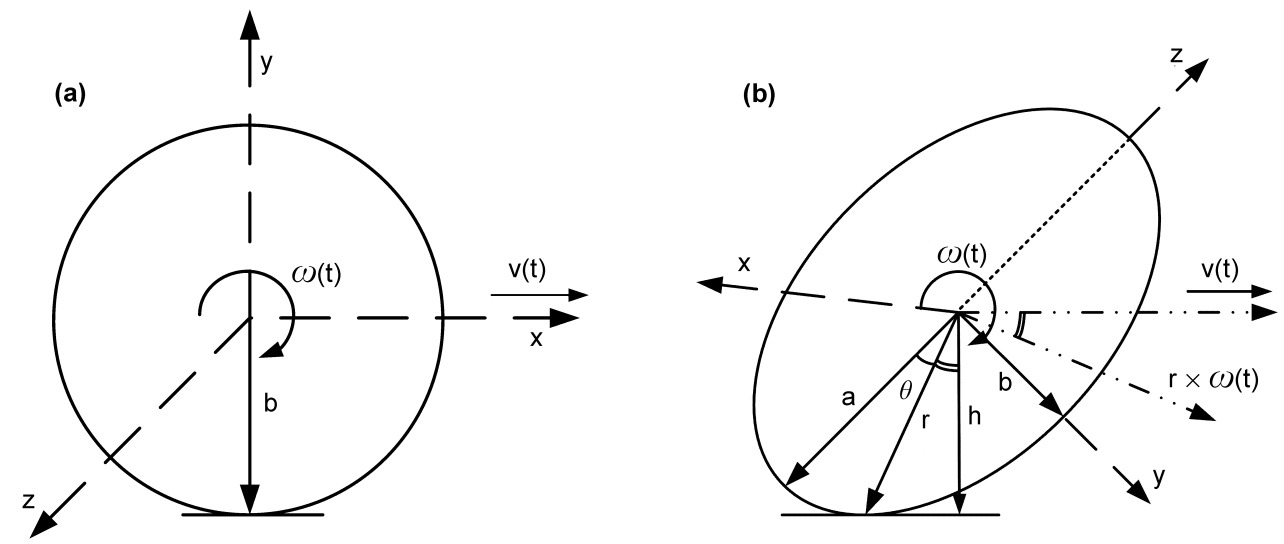

Figure 4. The ellipsoid from figure 3 is rotated (a) about the $z$-axis or (b) about the $x$-axis with translational velocity $v(t)$ and rotational velocity $\omega(t)$. In the figure, $r$ is the distance between the center of mass and the point of contact, $h$ is the perpendicular distance between the center of mass and the plane on which the ellipsoid is rolling, and $\theta$ is the angle between vectors $a$ and $r$.

The Lagrangian $L$ for the rolling motion of an ellipsoid with mass $m$ can be written as:

$$
L=\frac{1}{2} m v^{2}+\frac{1}{2} I \omega^{2}-m g h
$$

where $I$ is the moment of inertia about the axis of rotation, $h$ is the height of the center of mass from the plane on which the ellipsoid is rolling, $v$ is the translational velocity, and $\omega$ is the rotational velocity. Again, two cases were considered, the case where the ellipsoid rotates about the axially symmetric axis (the $z$-axis), and the case where it rotates about an axis perpendicular to the $z$-axis, e.g., the $x$-axis (fig. 4). The expressions for the inertial and geometric parameters are provided in table 3 .

As the goal of the action analysis was to acquire a better understanding of the orientation phenomena as an apple rolls down a test track, the acceleration used in the analysis was equated to the acceleration of an apple rolling down the test track, i.e.:

$$
\alpha=g \sin \gamma
$$

where $g$ is the acceleration due to gravity, and $\gamma$ is the slope of the track. For tests, a $\gamma$ value of $30^{\circ}$ is taken to be the standard condition. For simplicity, the model equations were derived assuming that ellipses roll on a level plane with constant acceleration. It would be possible to derive equations for a tilted plane; however, this effort is not warranted as the derivations would be more complicated and the predicted results would be essentially identical. The major difference between models for a level and a tilted plane involve potential energy, and potential energy components essentially cancel out in calculations of action differences. Thus, the translational velocity $v(t)$ is:

$$
\mathrm{v}(\mathrm{t})=\mathrm{v}_{0}+\alpha \mathrm{t}
$$

where $v_{0}$ is the initial velocity. The mass $m$ of the ellipsoid is:

$$
m=\rho \frac{4}{3} \pi a b^{2}
$$

where $\rho$ is the density. One of the major differences between the ellipsoid model and real apples is the density along the core of the apple. In real apples, the core of the apple is less dense than the remainder of the apple, and the concave nature of the stem and calyx further reduces the effective mass along the stem/calyx axis. However, while this difference might influence the magnitude of responses in subsequent analysis, the form of response curves should be conserved.

Table 3. Physical and dynamic parameters of the ellipsoid required for obtaining the action integral derived using the geometry of the ellipsoid in figure 3 . These parameters are calculated for two cases: rotation about the $z$-axis and rotation about an axis perpendicular to the $z$-axis, in this case the $x$-axis.

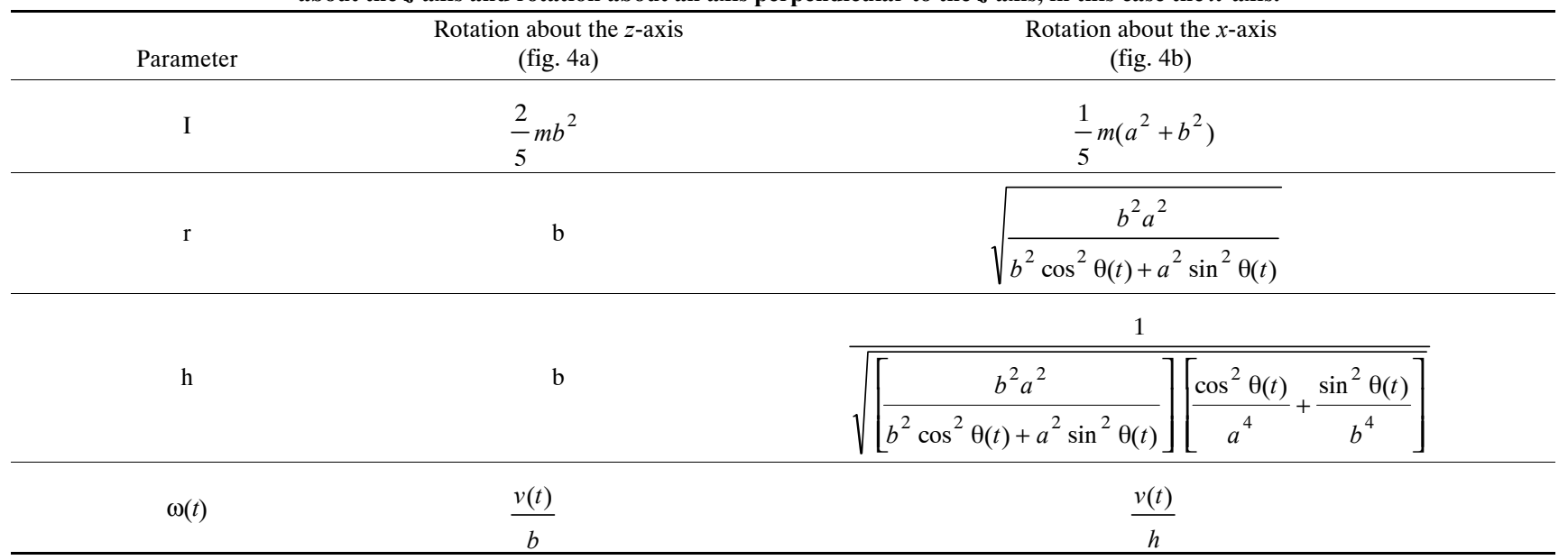


(a)

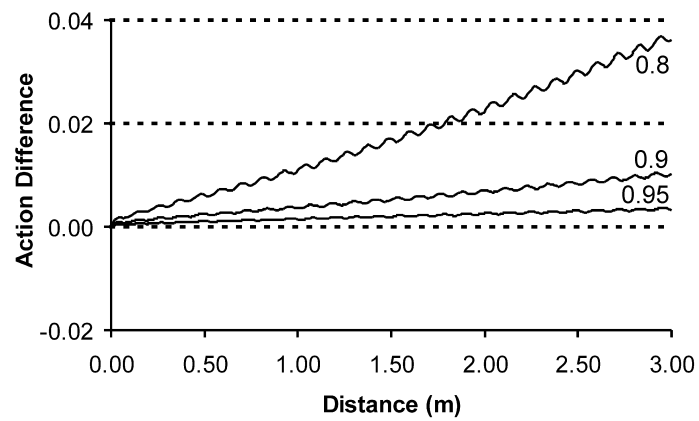

(b)

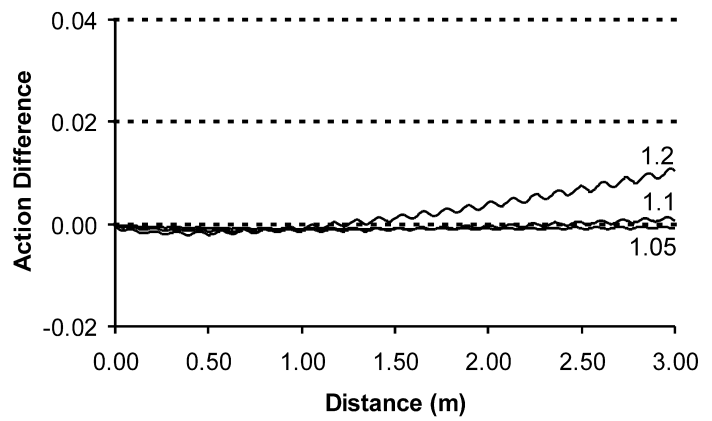

Figure 5. Action differences as a function of distance traveled for ellipsoids with constant volume (mass) and $a / b$ ratios (a) less than 1 or (b) greater than 1 . Note the initial negative action difference values for $a / b$ ratios greater than 1 and the variable distances traveled until the values exceed 0 .

By substituting the parameters from table 3 and using equations 21 and 22, the Lagrangian for the case when an object is rotating about the $z$-axis (fig. 4a) becomes:

$$
\begin{aligned}
L= & \frac{1}{2}\left(\rho \frac{4}{3} \pi a b^{2}\right)\left(v_{0}+\alpha t\right)^{2}+\frac{1}{5}\left(\rho \frac{4}{3} \pi a b^{2}\right) b^{2} \\
& \cdot \frac{\left(v_{0}+\alpha t\right)^{2}}{b^{2}}-\left(\rho \frac{4}{3} \pi a b^{2}\right) g b
\end{aligned}
$$

Similarly, the Lagrangian for the case when an object is rotating about the $x$-axis (fig. 4b) becomes:

\section{(a)}

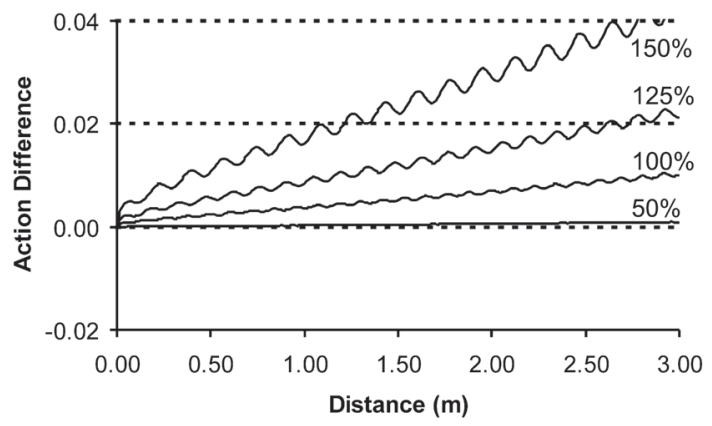

(c)

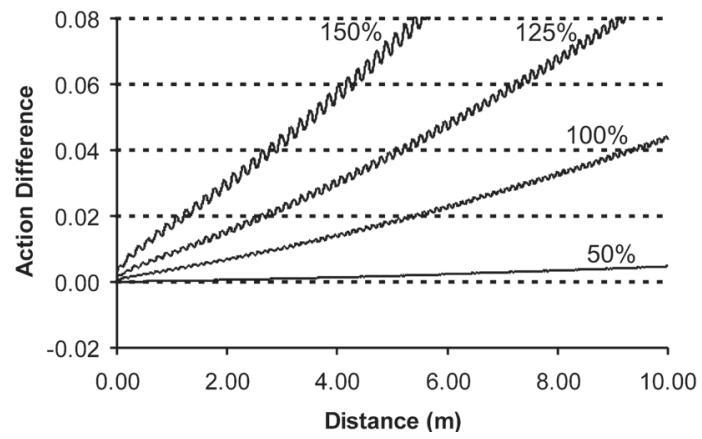

$$
\begin{aligned}
& L=\frac{1}{2}\left(\rho \frac{4}{3} \pi a b^{2}\right)\left(v_{0}+\alpha t\right)^{2}+\frac{1}{10}\left(\rho \frac{4}{3} \pi a b^{2}\right) \\
& \cdot\left(a^{2}+b^{2}\right)\left(v_{0}+\alpha t\right)^{2}\left[\frac{b^{2} a^{2}}{b^{2} \cos ^{2} \theta(t)+a^{2} \sin ^{2} \theta(t)}\right] \\
& \cdot\left[\frac{\cos ^{2} \theta(t)}{\left.a^{4}+\frac{\sin ^{2} \theta(t)}{b^{4}}\right]}\left[\frac{4}{3} \pi a b^{2}\right) g\right. \\
& -\frac{b^{2} a^{2}}{\sqrt{\left[\frac{b^{2} \cos ^{2} \theta(t)+a^{2} \sin ^{2} \theta(t)}{[}\right]\left[\frac{\cos ^{2} \theta(t)}{a^{4}}+\frac{\sin ^{2} \theta(t)}{b^{4}}\right]}}
\end{aligned}
$$

(b)

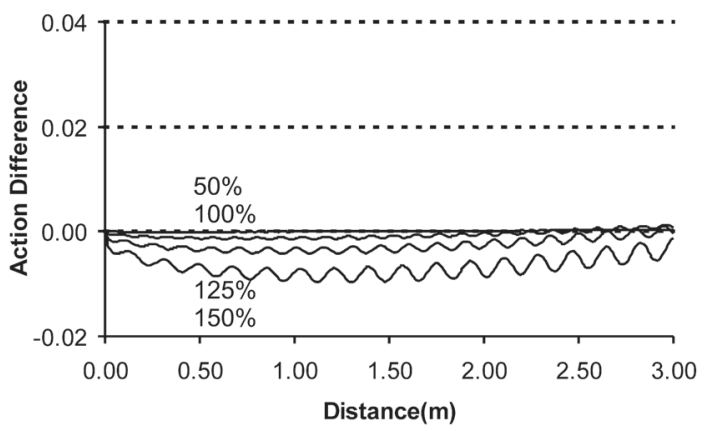

(d)

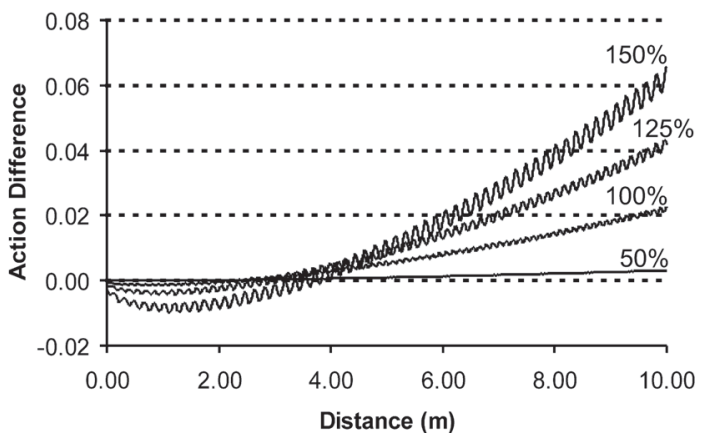

Figure 6. Action differences as a function of distance traveled for ellipsoids with the same shape and different volumes $(\mathrm{masses})$ for $(\mathrm{a}, \mathrm{c}) \mathrm{squat}(\mathrm{a} / \mathrm{b}=$ $0.9)$ and $(b, d)$ elongated $(a / b=1.1)$ ellipsoids. For ease of viewing, (a) and (b) show initial responses seen in (c) and (d) with magnified time scales. Note that for the elongated ellipsoid with $150 \%$ the mass of the standard ellipsoid, the distance traveled until the action difference crosses the distance axis is the longest. 
(a)

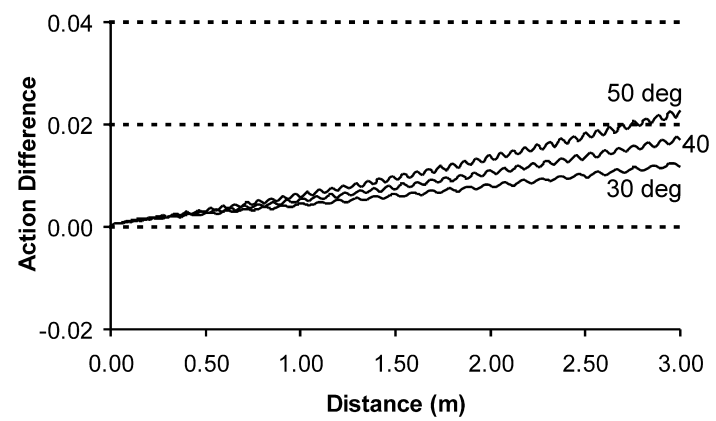

(b)

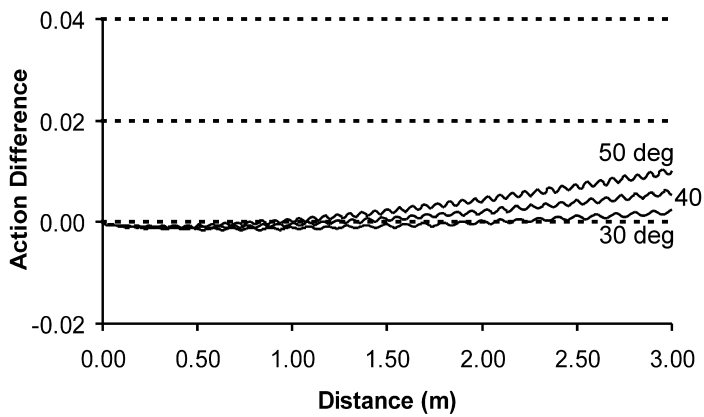

Figure 7. Action differences as a function of distance traveled with different levels of acceleration due to the angle of a theoretical test track for (a) squat $(a / b=0.9)$ or $(b)$ elongated $(a / b=1.1)$ ellipsoids. Note that the slopes of action differences increase with increasing acceleration.

The action difference for an ellipsoid was obtained by subtracting the action value for rotation about the $z$-axis from the corresponding values for rotation around the $x$-axis. Thus, a positive value for action difference implies a relative preference for rotation about the $z$-axis, and a negative value implies a relative preference for rotation about the $x$-axis.

\section{Stability of Ellipsoid Apple Model}

The first study examined the impact of the shape of the ellipsoid on the orientation process by keeping the volume constant while varying the $a / b$ ratio (fig. 5). For the case of squat apples $(a / b<1)$, the action difference is always positive and increasing. For the case of elongated apples $(a / b>1)$, the action difference initially is negative and has a negative slope. After some elapsed distance, the slope becomes positive, and the value increases until it crosses the distance axis and continues to increase. In general, the results indicate that as the shape of the ellipsoid approaches a sphere, the relative action differences are reduced as a function of

(a)

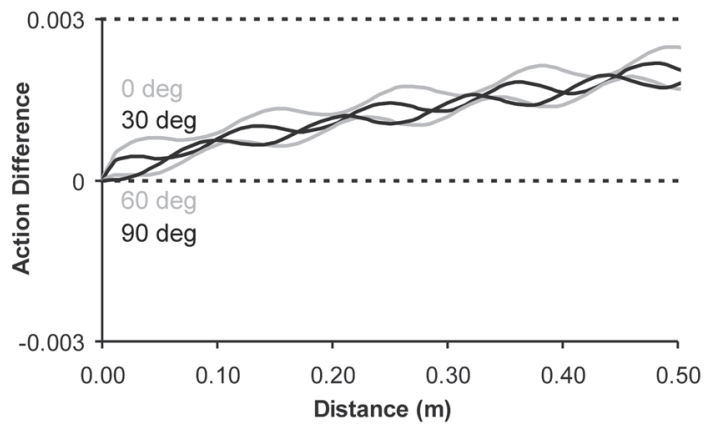

(c)

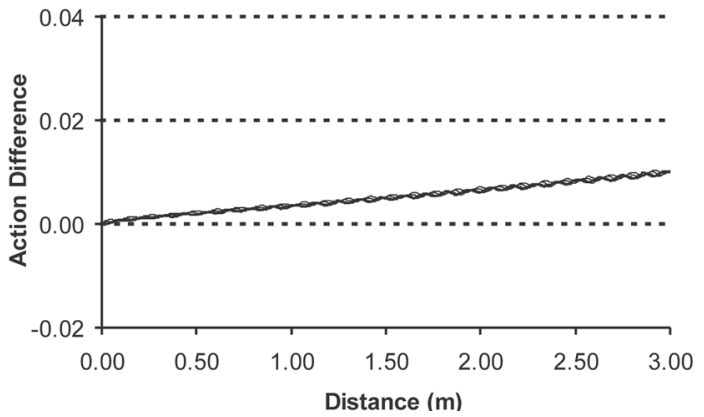

distance traveled. There are two practical conclusions that can be garnered from this study. First, it is hard to orient round apples using inertial properties. Second, the distance traveled until an elongated apple $(a / b>1)$ orients itself varies depending on shape. Both of these conclusions match preliminary observations of apples rolled down a test track (Narayanan et al., 2007).

The second study examined the effect of mass on the orientation process by varying the size (volume) of two different ellipsoid shapes while keeping density constant at $0.856 \mathrm{~g} \mathrm{~m}^{-3}$. An alternate test of the effect of mass on the orientation process might be to keep shape and size constant, and to vary mass. This alternative was not pursued as the mass of apples is fairly constant; thus, it would be difficult to test any predictions related to changes in density. However, the size of apples does vary. For this study, squat $(a / b=0.9)$ and elongated $(a / b=1.1)$ apples were tested using masses ranging from $50 \%$ to $150 \%$ of the standard apple mass (fig. 6). The

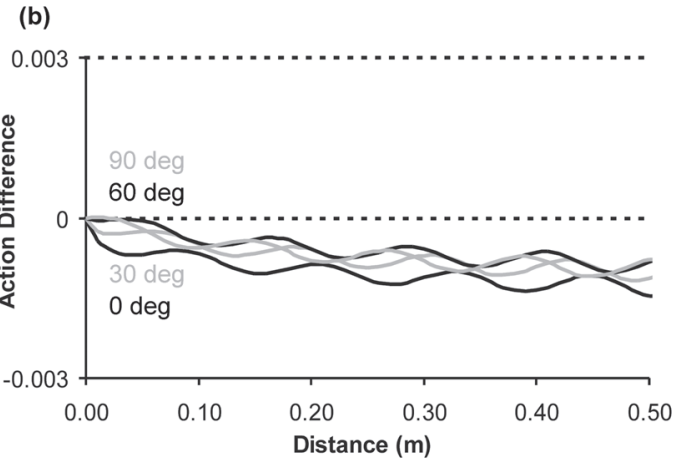

(d)

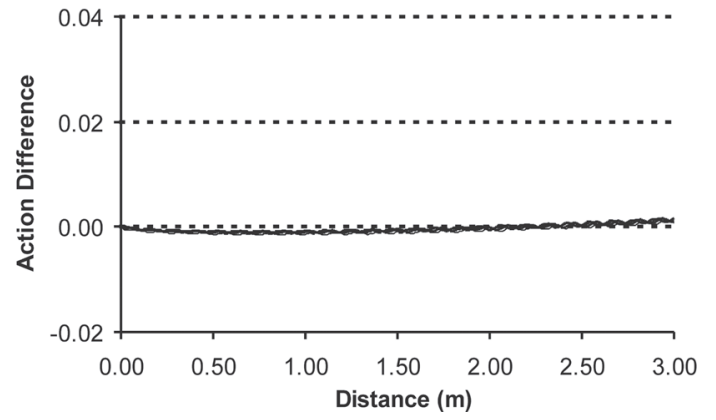

Figure 8. Action differences as a function of distance traveled for selected initial angels of rotation for $(a, c)$ squat $(a / b=0.9)$ and $(b, d)$ elongated $(a / b=$ 1.1) ellipsoids. For ease of viewing, (a) and (b) show initial responses seen in (c) and (d) with magnified time scales. 


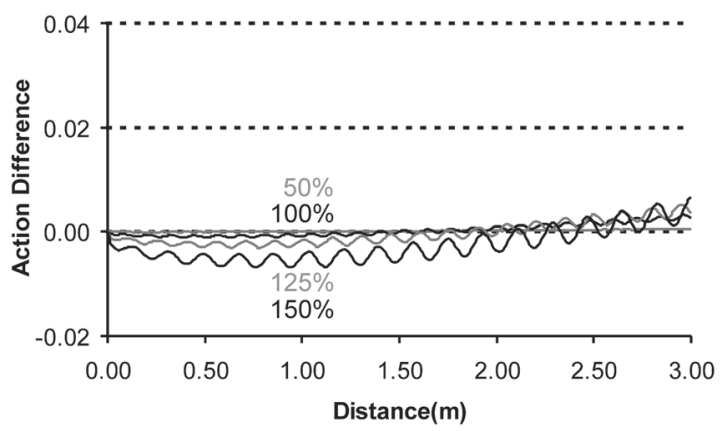

Figure 9. Action differences as a function of distance traveled for elongated $(a / b=1.1)$ ellipsoids of different masses when acceleration is increased by increasing the theoretical angle of a test track from the standard $30^{\circ}$ to $50^{\circ}$. Note the reduced distances before the action differences cross the distance axis compared to figure $6 \mathrm{~b}$, where the angle is $30^{\circ}$.

major challenge elucidated by this study is the finding that for elongated apples, the elapsed distance until the action difference becomes positive increases with increasing mass. The third study examined the effect of changing the acceleration constant by changing the theoretical angle of the test track (fig. 7). Increasing the acceleration increased the relative action difference as a function of distance traveled. Thus, for elongated apples, increasing acceleration reduced the relative travel distance necessary for the action difference to became positive. The fourth study addressed the effect of the initial value of angle $\theta$, which is the angular displacement (fig. 4b). Changing the angle altered the phase of the small oscillations, but did not alter the form of calculated action differences as a function of distance traveled (fig. 8).

The results of the above studies demonstrated that the shape and mass of elongated apples can have dramatic effects on the distance traveled until the action difference becomes positive. This variability raises a potential problem in regard to selecting a single location for imaging. The only tested factor that addresses this conundrum is the slope of the track, and a final study was conducted to directly test the effects of

(a)

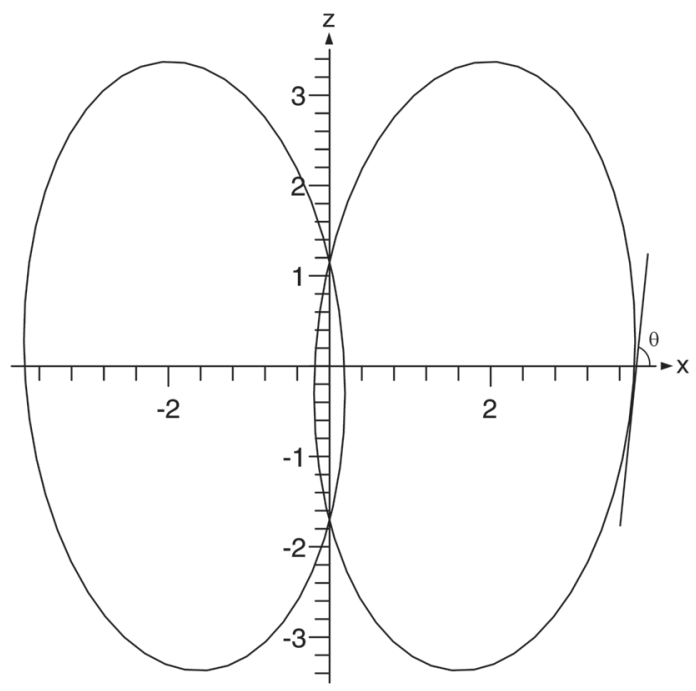

increased acceleration. As can be seen by comparing results in figure $6 \mathrm{~b}$ and figure 9 , increasing the theoretical track angle from $30^{\circ}$ to $50^{\circ}$ reduced the necessary travel distances until action differences become positive.

\section{Action Integral Using Spindle Torus Apple Model}

The Lagrangian $L$ for the rolling motion of a spindle torus apple model was determined using a method similar to that used for the ellipsoid model (eq. 19). The volume $V$ was calculated using equation 9 , and the moments of inertia were calculated using equations 11 and 12 . The major difference involves calculation of $h$, the height of the center of mass relative to the plane on which the spindle torus apple model is rolling. Equations for $h$ were derived for two cases: rotation about the axis of axial symmetry, and rotation about an axis perpendicular to this axis.

For the case where the apple is rolling on the axis of axially symmetry (z-axis), the height $h$ was calculated with respect to the largest diameter and was taken to be $D / 2$ (eq. 16). For the case where the apple is rolling on a perpendicular axis (e.g., the $x$-axis), the height $h$ was calculated with respect to the tangent line that goes around the apple, where $\theta$ varies from 0 to $2 \pi$ (fig. 10). For a given value of $\theta$, the parameter $u$ at the point of tangency $\left(u_{p}\right)$ was obtained using the following equation:

$$
\theta=\arctan \frac{x^{\prime}(u)}{y^{\prime}(u)}
$$

where $x^{\prime}(u)$ and $y^{\prime}(u)$ are obtained from equations 14 and 15 . The height $h$, which is the perpendicular distance between the origin (center of the apple) and the tangent, is calculated using the following equation:

$$
h=\sqrt{\left(x\left(u_{p}\right)+s x^{\prime}\left(u_{p}\right)\right)^{2}+\left(y\left(u_{p}\right)+s y^{\prime}\left(u_{p}\right)\right)^{2}}
$$

where

$$
s=\frac{-x\left(u_{p}\right) x^{\prime}\left(u_{p}\right)-y\left(u_{p}\right) y^{\prime}\left(u_{p}\right)}{x^{\prime}\left(u_{p}\right)^{2}+y^{\prime}\left(u_{p}\right)^{2}}
$$

(b)

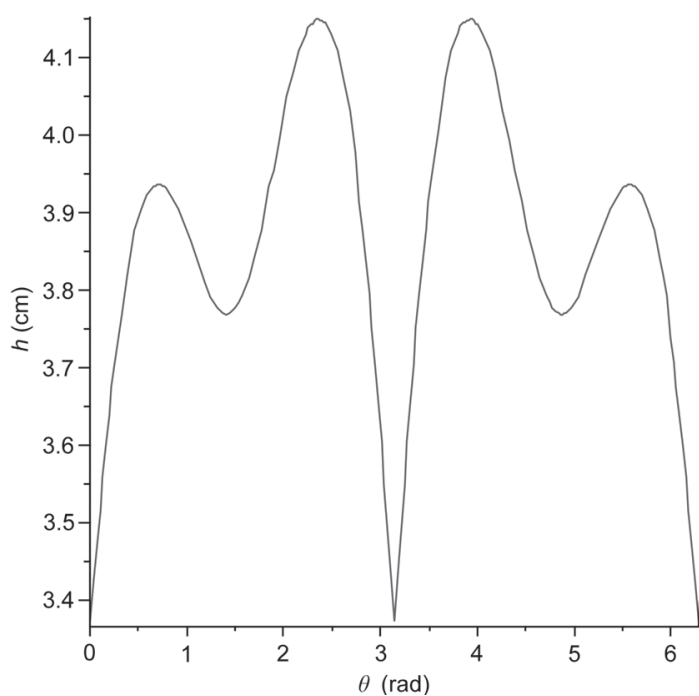

Figure 10. Apple model generated for the Braeburn cultivar $(a=3.38 \mathrm{~cm}, b=1.98 \mathrm{~cm}, c=1.80 \mathrm{~cm}, T=1.49 \mathrm{rad})$. In (a), note the tangent line that is used to calculate height $h$. The plot of $h$ as $\theta$ varies from 0 to $2 \pi$ is shown in (b). 
(a)

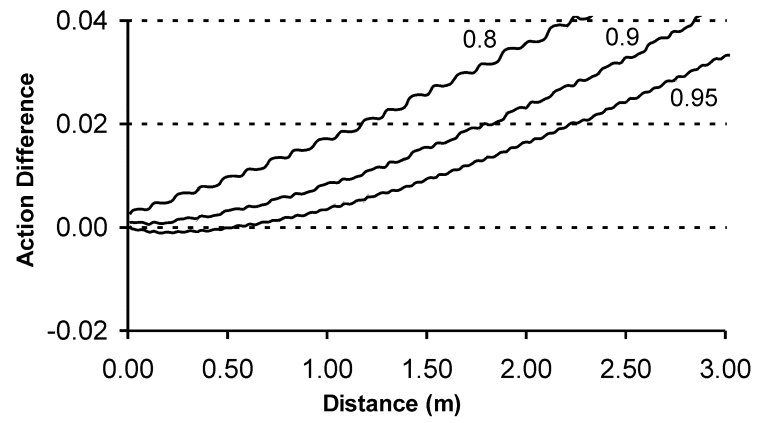

(b)

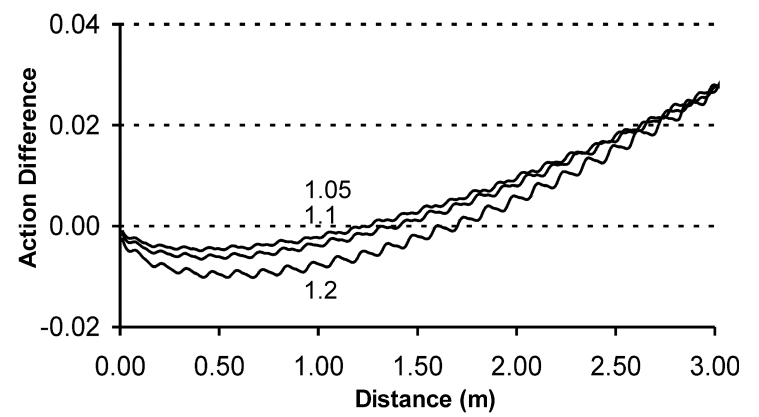

Figure 11. Action differences as a function of distance traveled for spindle torus apple models with constant volume (mass) and $L E / D$ ratios (a) less than 1 or (b) greater than 1 . For ease of comparison, graphs are scaled the same as in figure 5.

(a)

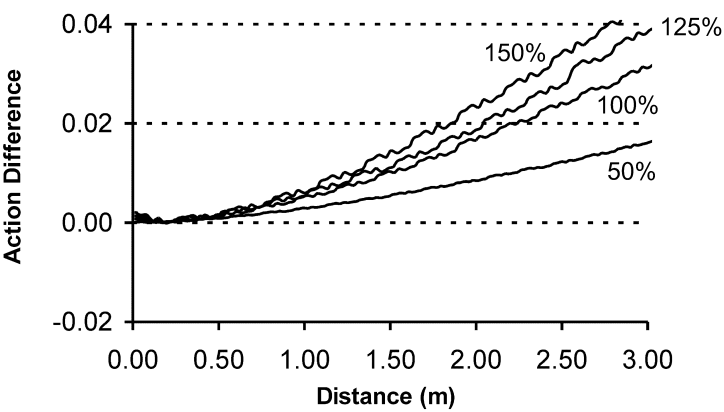

(b)

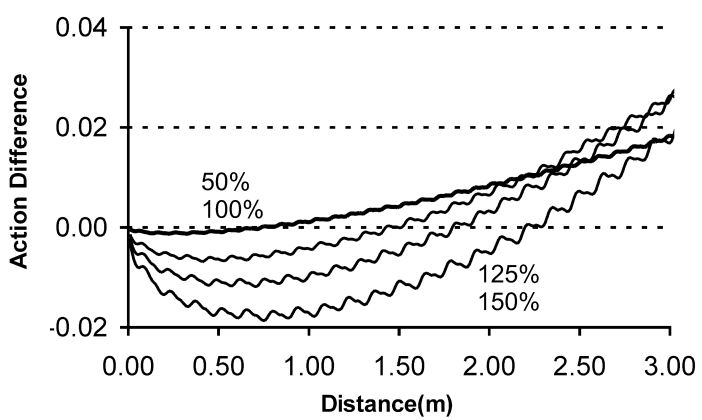

Figure 12. Action differences as a function of distance traveled for spindle torus apple models with the same shape and different volumes (masses) for (a) "Braeburn" $(L E / D=0.9)$ and $(b)$ "Red Delicious" $(L E / D=1.1)$ apples. For ease of comparison, graphs are scaled the same as in figure 6.

Table 4. Spindle torus apple model parameters for apples tested in figure 11 and 12 . For figure 12, volumes were altered by multiplying $a, b$, and $c$ values by a constant while keeping $\phi$ the same.

\begin{tabular}{lccccc}
\hline Apple Cultivar & $\begin{array}{c}\text { LE/D } \\
\text { Ratio }\end{array}$ & $\begin{array}{c}\mathrm{a} \\
(\mathrm{cm})\end{array}$ & $\begin{array}{c}\mathrm{b} \\
(\mathrm{cm})\end{array}$ & $\begin{array}{c}\mathrm{c} \\
(\mathrm{cm})\end{array}$ & $\begin{array}{c}\phi \\
(\mathrm{rad})\end{array}$ \\
\hline Red Delicious & 1.2 & 4.12 & 2.20 & 1.23 & 1.55 \\
Red Delicious & 1.1 & 3.86 & 2.20 & 1.31 & 1.55 \\
Red Delicious & 1.05 & 3.72 & 2.15 & 1.39 & 1.55 \\
Red Delicious & 0.95 & 3.48 & 2.21 & 1.45 & 1.55 \\
Braeburn & 0.9 & 3.37 & 2.30 & 1.43 & 1.49 \\
Granny Smith & 0.8 & 3.13 & 2.42 & 1.47 & 1.46 \\
\hline
\end{tabular}

\section{Stability of Spindle Torus Model}

Results for the apple torus models were very similar in form to results for the ellipsoid apple models. The primary difference was a greater tendency to prefer rotation about the axis of axial symmetry. For the case of constant volume with different $L E / D$ ratios, comparison of figure 11 to figure 5 shows that positive slopes of action differences were greater for the spindle torus apple models, and that the transition from negative to positive action differences for elongated "apples" occurred earlier and more dramatically. For the case of constant shapes with different volumes, comparison of figure 12 to figure 6 again shows that positive slopes of action differences were greater for the spindle torus apple models and, for elongated apples, the distances traveled before the action differences became positive were much shorter, approximately $2 \mathrm{~m}$ for the spindle torus apples versus $4 \mathrm{~m}$ for the ellipsoid apples. The parameters used to generate the spindle torus models of apples in figures 11 and 12 are listed in table 4 . While the spindle torus results are probably more realistic, as the spindle torus "apples" more accurately reflect the shape and the reduced density at the core of real apples compared to ellipsoid "apples", the ellipsoid model is easier to deal with both in formulation and computation. This ease of application suggests that the simpler model may be preferable for initial analyses. For brevity, figures 7 to 9 are not replicated. Again, results were similar in form. The initial angle of inclination has little influence on calculated action differences, and the relatively short distances needed for worst-case action differences to become positive negates the need to examine effects of acceleration.

\section{Comparison of Results to Prior Expectations}

Previous literature states that it is easier to orient elongated cultivars of apples (Whitelock et al., 2006). Intuitively this conclusion makes sense; if elongated apples and squat apples are placed calyx-down on a flat table and the table is jostled, the elongated apples are much more likely to fall over. Thus, the initial assumption was that it would be easy to orient elongated apples, but that it would be hard to orient squat apples. The results of the analysis using action integrals suggested just the opposite. Preliminary tests with squat apples demonstrated that most such apples oriented themselves almost immediately after starting to roll down the test track (Narayanan et al., 2007). It was this result that inspired us to expand analysis using action integrals. A particular preliminary observation that bothered us was the unexplained variability in the distance that apples traveled down the test track before changing to an oriented posture. The action integral analysis suggests that this variability is due to the shape and mass of the apples. With this knowledge, 
it may be possible design and test sorting and processing systems that minimize effects of this variability.

\section{Implications}

Theoretical analyses suggest that it may be possible to develop a practical orientation system based on inertial properties of fruits. However, there are a number of potential complications raised by this study that need to be addressed. From the analysis, the important factors that can potentially affect the orientation process were found to be the mass and $L E / D$ ratio of an apple. These factors affect the distance an "apple" has to travel before it becomes oriented. For a commercial inspection system, the imaging camera has to be positioned at a fixed location, and the fruit have to be oriented prior to the camera location. Results suggest that the distance problem can be addressed by varying the slope of the tracks or pre-sorting the fruit by size or shape. However, varying the slope could cause technical difficulties, as steeper tracks would increase the terminal velocity of the rolling fruit, which could then create problems for unloading the fruit from the tracks. To overcome the problem with terminal velocity, tracks with varying slope or curved tracks could be considered. Another possibility is a system with a pre-sorting unit that feeds sets of tracks with different slopes. Thus, the complications identified by the analysis can potentially be addressed by using some combination of these design methodologies.

The analytic method using the action integral produced results that help explain the orientation phenomena and also provide insight for design of future experiments. Results were similar in form for both ellipsoid and spindle torus apple models. The demonstrated value of these results suggests that the method may be useful for analysis of other dynamic problems. Typically, developing an analytical model to test the dynamics of a system involves complex mathematics and often requires the inclusion of various factors, such as friction, reaction forces, etc. The approach described in this article looks promising as an effective and simple tool for the preliminary analysis of a dynamic system. Analysis based on the action integral may be helpful in several ways: First, by providing insight for the development of more complex dynamic models. Second, in deciding whether it is worthwhile to develop the model, i.e., in determining if the effort to develop a more complex system model is justified. Finally, the action integral analysis might provide sufficient understanding of the dynamic system so that more detailed analysis would not be necessary.

\section{Caveats}

The models described in the article were developed to investigate stability questions and they are not rigorous dynamic models. For example, the models do not include factors such as contact or friction forces, and these factors play a major role in the orientation process. The models also do not consider that test tracks have two rails and thus two points of contact for apples rolling down the tracks. As a consequence, it is inappropriate to directly compare model predictions with empirical test results. The validity of the models can best be examined by comparing the qualitative predictions of the role of factors such as mass and size on the orientation process with empirical results.

More specifically, the stability of a freely spinning rigid body may have limited implications for an apple rolling down a test track, as there is no consideration of the reaction forces against the tracks. In addition, the validity of the generalized stability equation derived using the small perturbation method has been debated. However, more rigorous derivations yield comparable results (Meirovitch, 1988).

Similarly, the interpretation of the results for analysis using the action integral depends on a number of assumptions. The essential assumption is that for the same object traveling the same path with different initial orientations, lower action values represent preferred motions. For ease of analysis, a functional assumption was made that acceleration was constant. For rotation around the non-axially symmetric axis, this assumption results in motions that are not physically possible. The assumption was made so that the distance traveled over a given time would be consistent for both cases studied. With a physically valid model, conservation of momentum would have to be considered, and the effective rate of travel would be reduced for the situation of rotation around the non-axially symmetric axis. It is not clear, under these conditions, whether the values used to calculate the action difference should then be based on elapsed time or physical location.

For comparison of action differences, the time of transition to a positive action difference was implicitly assumed to be the time at which an "apple" would switch to an "oriented" position. A more realistic assumption might have been to use some positive threshold rather than the zero crossing. A positive threshold would acknowledge the practical consideration that some energy difference must exist before a transition can be accomplished. However, interpretation of results was done only on a relative basis, so use of a positive threshold would have little effect.

While there is no theoretical justification for many of the assumptions, the predicted results are intuitively reasonable, many of the results agree with preliminary observations (Narayanan et al., 2007), and there are no predicted results that fly in the face of reality. The true value of the analysis is that it makes predictions that can be tested experimentally.

\section{Comparison of Predictions to Experimental Results}

The results of this study were used to design empirical tests of the factors that were identified as having a potential influence on the distance an apple travels prior to moving to an oriented position (Narayanan et al., 2007). In agreement with predictions, initial orientation was found to be by far the most important factor governing the distance an apple travels prior to orientation. The next most important factor was the mass of the apple; orientation distance decreased as mass increased. Additionally, there was some evidence that orientation distance increased as the length-to-diameter ratio approached one. However, except for the effect of initial orientation, the impact of factors suspected of influencing orientation based on the theoretical model predictions were obscured by the influence of factors such as precession and slippage. This result only further emphasizes the fact that the theoretical models should not be used in an attempt to model the dynamics of the orientation process. That the empirical results are qualitatively consistent with theoretical predictions validates the contention that the primary factor underlying the orientation process is the inertial characteristics of apples. 


\section{Conclusion}

The question addressed by this study was whether stability analysis could help explain why apples, after achieving sufficient angular velocity, orient themselves to rotate around the stem/calyx axis. It was hypothesized that an apple could be considered to be an object with axial symmetry, and that stability criteria based on inertial characteristics of an axially symmetric object could be used to explain this phenomena. The results of stability analysis of a free body with an axially symmetric axis demonstrated that rotation around that axis is stable, while rotation around a perpendicular axis is not. More detailed analysis using the action integral elucidated conditions in which rotation around the axially symmetric axis was preferred to rotation around a perpendicular axis. For this analysis, ellipsoids of selected sizes and shapes were assumed to roll in a straight line under constant acceleration. A critical assumption for interpretation of the results was that for the same object traveling the same path with different initial orientations, lower action values represent preferred motions. In general, rotation around the axially symmetric axis was found to be the preferred motion. Of practical interest are the findings that both the mass and the shape of "apples" affect the distance that an apple must travel before oriented motion becomes the preferred motion. Thus, this study demonstrates the value of a novel use of action integrals to examine stability and provides justification for efforts to develop a practical system for orienting fruit based on rotational stability.

\section{REFERENCES}

Bennedsen, B. S., and D. L. Peterson. 2004. Identification of apple stem and calyx using unsupervised feature extraction. Trans. ASAE 47(3): 889-894.

Bennedsen, B. S., and D. L. Peterson. 2005. Performance of a system for apple surface defect identification in near-infrared images. Biosystems Eng. 90(4): 419-431.

Brosnan, T., and D. W. Sun. 2004. Improving quality inspection of food products by computer vision: A review. J. Food Eng. 61(1): 3-16.

Brown, G. K., L. J. Segerlind, and R. Summitt. 1974. Near-infrared reflectance of bruised apples. Trans. ASAE 17(1): 17-19.

Brown, I. A., E. P. Ristow, and R. D. Walters. 1988. Roller for orientation of fruit. U.S. Patent No. 4,730,719. Washington, D.C.: Commissioner of Patents and Trademarks.

Brusewitz, G. H., M. P. Rigney, and M. L. Stone. 1995. Mechanical orienter for on-line sorting of peaches. In Proc. Food Processing Automation IV Conf., 451-460. Chicago, Ill.: Food and Process Engineering Institute.

Chen, Y. R., K. Chao, and M. S. Kim. 2002. Machine vision technology for agricultural applications. Computers and Electronics in Agric. 33(2/3): 173-191.

Greenwood, D. T. 1965. Principles of Dynamics. Englewood Cliffs, N. J.: Prentice-Hall.

Kim, M. S., A. M. Lefcourt, Y. R. Chen, I. Kim, D. E. Chan, and K. Chao. 2002. Multispectral detection of fecal contamination on apples based on hyperspectral imagery: Part II. Application of hyperspectral fluorescence imaging. Trans. ASAE 45(6): 2039-2047.
Kleynen, O., V. Leemans, and M. F. Destain. 2005. Development of a multi-spectral vision system for the detection of defects on apples. J. Food Eng. 69(1): 41-49.

Lefcourt, A. M., M. S. Kim, and Y. R. Chen. 2003. Automated detection of fecal contamination of apples by multispectral laser-induced fluorescence imaging. Applied Optics 42(19): 3935-3943.

Lefcourt, A. M., M. S. Kim, and Y. R. Chen. 2005. Detection of fecal contamination on apples with nanosecond-scale time-resolved imaging of laser-induced fluorescence. Applied Optics 44(7): 1160-1170.

Li, Q. Z., M. H. Wang, and W. K. Gu. 2002. Computer vision based system for apple surface defect detection. Computers and Electronics in Agric. 36(2-3): 215-223.

Marion, J. B. 1965. Classical Dynamics of Particles and Systems. London, U.K.: Academic Press.

Megilley, B. W., H. P. Rasmussen, and D. H. Dewey. 1968. Fruit characteristics affecting apple orientation in water. Michigan Quarterly Bulletin 50(4): 527-537.

Mehl, P. M., Y. R. Chen, M. S. Kim, and D. E. Chan. 2004. Development of hyperspectral imaging technique for the detection of apple surface defects and contaminations. J. Food Eng. 61(1): 67-81.

Meirovitch, L. 1988. Methods of Analytical Dynamics. New York, N.Y.: McGraw-Hill.

Narayanan, P., A. M. Lefcourt, U. Tasch, R. Rostamian, and M. S. Kim. 2007. Tests of the ability to orient apples using their inertial properties. In Proc. ASABE Annual International Meeting. ASABE Paper No. 07646. St. Joseph, Mich.: ASABE.

Penman, D. W. 2001. Determination of stem and calyx location on apples using visual inspection. Computers and Electronics in Agric. 33(1): 7-18.

Rigney, M. P., G. H. Brusewitz, and M. L. Stone. 1996. Peach physical characteristics for orientation. Trans. ASAE 39(4): 1493-1497.

Stout, B. A., D. H. Dewey, and R. F. Mrozek. 1971. Mechanical orientation of apples and related fruit characteristics. Michigan Agric. Exp. Sta. Res. Bull. 32 (Aug. 1971).

Tasch, U., A. Grinblat, P. Narayanan, A. M. Lefcourt, and M. S. Kim. 2006. Apparatus and method for orienting rotatable objects. Pending U.S. Patent Application. 6 June 2006.

Throop, J. A., D. J. Aneshansley, and B. L. Upchurch. 1999. Fruit orienting device. U.S. Patent No. 5,588,270. Washington, D.C.: Commissioner of Patents and Trademarks.

Throop, J. A., D. J. Aneshansley, B. L. Upchurch, and B. Anger. 2001. Apple orientation on two conveyors: Performance and predictability based on fruit shape characteristics. Trans. ASAE 44(1): 99-109.

Throop, J. A., D. J. Aneshansley, W. C. Anger, and D. L. Peterson. 2005. Quality evaluation of apples based on surface defects: Development of an automated inspection system. Postharvest Biol. and Tech. 36(3): 281-290.

Wen, Z., and Y. Tao. 2000. Dual-camera NIR/MIR imaging for stem-end/calyx identification in apple defect sorting. Trans. $A S A E$ 43(2): 446-452.

Whitelock, D. P., G. H. Brusewitz, and M. L. Stone. 2006. Apple shape and rolling orientation. Applied Eng. in Agric. 22(1): 87-94. 